\title{
Fasting Hyperglycemia Increases In-Hospital Mortality Risk in Nondiabetic Female Patients with Acute Myocardial Infarction: A Retrospective Study
}

\author{
Guojing Luo, Hong Liu, Shunkui Luo, Fang Li, Minhong Su, and Hongyun Lu \\ Department of Endocrinology and Metabolism, the Fifth Affiliated Hospital of Sun Yat-sen University, Zhuhai, \\ Guangdong 519000, China
}

Correspondence should be addressed to Hongyun Lu; luhongyun2013@163.com

Received 18 April 2014; Revised 14 June 2014; Accepted 19 June 2014; Published 14 July 2014

Academic Editor: Alexandra Kautzky-Willer

Copyright (C) 2014 Guojing Luo et al. This is an open access article distributed under the Creative Commons Attribution License, which permits unrestricted use, distribution, and reproduction in any medium, provided the original work is properly cited.

\begin{abstract}
Previous studies had shown that elevated admission plasma glucose (APG) could increase mortality rate and serious complications of acute myocardial infarction (AMI), but whether fasting plasma glucose (FPG) had the same role remains controversial. In this retrospective study, 253 cases of AMI patients were divided into diabetic $(n=87)$ and nondiabetic group $(n=166)$. Our results showed that: compared with the nondiabetic patients, diabetic patients had higher APG, FPG, higher plasma triglyceride, higher rates of painless AMI $(P<0.01)$, non-ST-segment elevation myocardial infarction (NSTEMI), and reinfraction $(P<0.05)$. They also had lower high density lipoprotein cholesterol and rate of malignant arrhythmia, but in-hospital mortality rate did not differ significantly $(P>0.05)$. While nondiabetic patients were subgrouped in terms of APG and FPG (cut points were $11.1 \mathrm{mmol} / \mathrm{L}$ and $7.0 \mathrm{mmol} / \mathrm{L}$, resp.), the mortality rate had significant difference $(P<0.01)$, whereas glucose level lost significance in diabetic group. Multivariate logistic regression analysis showed that FPG (OR: 2.014; 95\% confidence interval: 1.296-3.131; $p<0.01$ ) but not APG was independent predictor of in-hospital mortality for nondiabetic patients. These results indicate that FPG can be an independent predictor for mortality in nondiabetic female patients with AMI.
\end{abstract}

\section{Introduction}

Incidence of AMI in female patients is increasing year by year after menopause, especially for type 2 diabetes mellitus (T2DM) patients. Gender disparity in clinical outcome of AMI patients with or without T2DM is still elusive. Women with AMI are more inclined to gain a poorer outcome than men [1-3]. Plasma glucose is often considered as an important predictor of mortality after AMI [3-6]. But the impact of fasting plasma glucose (FPG) on early mortality and serious cardiovascular complications such as malignant arrhythmia, cardiac shock, heart failure, and reinfarction remains unclear.

Plasma glucose level in the acute phase of AMI is closely related to in-hospital mortality rate and serious complications of AMI. Previous studies have demonstrated that there is a near-linear positive relationship between admission plasma glucose (APG) or HbAlc and in-hospital mortality in diabetic and nondiabetic AMI patients $[7,8]$. However, other studies showed that the relationship is u-shaped in AMI patients [9-11]. Moreover, previous studies mainly focused on the relationship between APG and clinical outcome. Limited data is available for association between FPG and clinical outcome of AMI with or without T2DM in female patients.

To assess the relationship between FPG and AMI prognosis in female patients, we conducted this retrospective analysis to determine the association between APG, FPG, and serious cardiovascular complications of female AMI patients with or without diabetes.

\section{Patients and Methods}

2.1. Subjects and Diagnostic Criteria. From January 2002 to February 2014, a total of 253 cases of consecutive female patients who were admitted to the Fifth Affiliated Hospital of Sun Yat-sen University in China with their first AMI 
diagnosis were enrolled into the retrospective study. AMI was defined by the following characteristics: chest pain consistent with ongoing myocardial ischemia persisting $>30$ minutes, ischemic electrocardiographic changes, and positive biochemical cardiac necrosis markers measurement (peak creatinine kinase value $>2$ times the normal upper limit or elevation of serum troponin $\mathrm{I}(\mathrm{cTnI})$ or serum troponin $\mathrm{T}(\mathrm{cTnT}))$. STEMI was diagnosed if ST-segment elevation $\geq 1 \mathrm{~mm}$ occurred in $\geq 1$ lead or new left bundle branch block (LBBB) was found in ECG with biochemical evidence of myocardial necrosis. NSTEMI was diagnosed in patients with $\geq 1$ positive biochemical cardiac necrosis markers measurement without new ST-segment elevation in ECG. Malignant arrhythmia is defined as fast or slow arrhythmia that significantly influences blood flow dynamics, including ventricular tachycardia, ventricular flutter, ventricular fibrillation, threedegree AVB, and fast atrial fibrillation with unstable hemodynamic. Cardiogenic shock was defined as reduced blood pressure $(\mathrm{SBP}<90 \mathrm{mmHg}$ or a drop of mean arterial pressure $>$ $30 \mathrm{mmHg})$ and/or low urine output $(<0.5 \mathrm{~mL} / \mathrm{kg} / \mathrm{h})$, with a pulse rate $>60 \mathrm{bpm}$ with or without evidence of organ congestion [12].

Enrolled patients were divided into diabetic group and nondiabetic group based on their final diagnosis [13]. Patients were thought to have diabetes if they had a previous or current diagnosis of diabetes, regardless of glycemic status on admission. The exclusion of T2DM was confirmed by the measurement of nonfasting glucose and fasting glucose before discharge. Each group was divided into two prespecified groups based on APG level $(<11.1$ and $\geq 11.1 \mathrm{mmol} / \mathrm{L}$ ) and FPG level $(<7.0$ and $\geq 7.0 \mathrm{mmol} / \mathrm{L})$. And we defined the former (APG $<11.1 \mathrm{mmol} / \mathrm{L}$ and $\mathrm{FPG}<7.0 \mathrm{mmol} / \mathrm{L}$ ) as nonhyperglycemia subgroup and the latter as hyperglycemia subgroup. This study excluded patients with a history of malignant tumor, chronic renal failure (creatinine > $451 \mu \mathrm{mol} / \mathrm{L}$ ), liver cirrhosis, serious infected diseases, and previous myocardial infarction.

2.2. Clinical Data Collection. Clinical symptoms and signs including chest pain or painless, systolic blood pressure (SBP), diastolic blood pressure (DBP), and heart rate (HR) were recorded on admission. Blood samples, including APG, creatinine, and creatinine kinase (CK), were measured at the time of hospital admission. FPG, total cholesterol (TC), triglyceride (TG), high-density lipoprotein cholesterol (HDL-C), and low-density lipoprotein cholesterol (LDL-C) were measured in the next day's morning after overnight fasting. According to the results of electrocardiograph (ECG), they were classified into non-ST-segment elevation myocardial infarction (NSTEMI) or ST-segment elevation myocardial infarction (STEMI). Killip class was used for the assessment of the severity of heart failure. The primary end point was all-cause in-hospital mortality; the second end points were serious cardiovascular complications such as malignant arrhythmia, cardiac shock, heart failure, and reinfarction.

2.3. Statistical Analysis. Continuous variables are expressed as mean \pm SD or median \pm interquartile range, and categorical variables were reported as numbers and percentages. Statistical analysis was performed with chi-square test for categorical variables. The $t$-test was used for continuous variables. Logistic regression analyses were used to determine the predictors of in-hospital mortality. In order to account for the influence of the risk factors on mortality rate, we performed a enter regression analysis with in-hospital death as outcome and the other risk factors (age, APG, FPG, SBP, DBP and HR at admission, blood lipid, and creatinine) as covariates. All 2sided $P$ values $<0.05$ were considered statistically significant. Analyses were done using the statistical software SPSS 13.0.

\section{Results}

3.1. Baseline Clinical Characteristics. From February 2002 to February 2014, a total of 253 female patients with their first AMI diagnosis were enrolled in this study. Diabetic group included 87 patients (34\%) and nondiabetic group included 166 patients $(66 \%)$. They had average ages of $70.11 \pm 9.80$ and $70.32 \pm 12.30$ years old, respectively. There was no difference in the age between the two groups, as shown in Figure 2.

Table 1 presents the baseline clinical characteristics of patients with or without diabetes. There was significant difference in APG and FPG between the two groups. Mean APG was significantly higher in diabetic patients than nondiabetic patients $(13.9 \pm 6.2$ versus $8.5 \pm 3.7 \mathrm{mmol} / \mathrm{L}, P<0.01)$. Besides, mean FPG was also remarkably higher in diabetic patients $(9.1 \pm 3.5$ versus $5.9 \pm 1.3 \mathrm{mmol} / \mathrm{L}, P<0.01)$. For the blood lipid profile, plasma triglyceride level was obviously higher and HDL-C was lower in diabetic group. Compared with the nondiabetic group, diabetic patients were more likely to have atypical clinical presentations of AMI. The proportions of painless AMI and NSTEMI were statistically higher among diabetic patients than the nondiabetic group. In addition, diabetic patients may have a greater reinfarction rate after the first AMI than nondiabetic patients $(13.79 \%$ versus $6.02 \%, P<0.05)$. However, the incidence rate of malignant arrhythmia in diabetes group was lower than nondiabetic group (2.3\% versus $11.45 \%, P<0.05)$.

\subsection{Relationship of APG and FPG to Serious Complications of} $A M I$. The association between different blood glucose level (APG and FPG) and clinical characteristics and complications of AMI was listed in Tables 2 (two missing values for FPG in non-diabetic group) and 3 (11 missing values for FPG in non-diabetic group and six in diabetic group). A total of 34 deaths (13.44\%) occurred during hospital stay in two groups (Table 1). In-hospital mortality of diabetic group did not differ significantly from nondiabetic group $(16.09 \%$ versus $12.05 \%, P=0.37)$. But the mortality rate of nonhyperglycemia subgroup (APG $<11.1 \mathrm{mmol} / \mathrm{L}, \mathrm{FPG}<7.0 \mathrm{mmol} / \mathrm{L}$ ) and hyperglycemia subgroup $(\mathrm{APG} \geq 11.1 \mathrm{mmol} / \mathrm{L}, \mathrm{FPG} \geq$ $7.0 \mathrm{mmol} / \mathrm{L}$ ) was dramatically different in the nondiabetic group (Tables 2 and 3). There was a tendency towards a much higher in-hospital mortality rate in nondiabetic group with the rising APG level and FPG level (Figure 1). When nondiabetic patients were subgrouped by APG level, the inhospital mortality rate in nonhyperglycemia subgroup and 
TABLE 1: Baseline characteristics of patients with and without diabetes mellitus.

\begin{tabular}{|c|c|c|c|}
\hline Variable & Nondiabetic group & Diabetic group & $P$ value \\
\hline Cases & 166 & 87 & \\
\hline Age (years) & $70.32 \pm 12.30$ & $70.11 \pm 9.80$ & 0.88 \\
\hline Hypertensions & $98(59.03 \%)$ & $61(70.11 \%)$ & 0.08 \\
\hline Painless AMI & $35(21.08 \%)$ & $30(34.48 \%)$ & 0.02 \\
\hline HR (bpm) & $83 \pm 23$ & $86 \pm 21$ & 0.43 \\
\hline $\mathrm{SBP}(\mathrm{mmHg})$ & $135 \pm 30$ & $140 \pm 28$ & 0.19 \\
\hline DBP (mmHg) & $82 \pm 20$ & $80 \pm 14$ & 0.34 \\
\hline APG (mmol/L) & $8.50 \pm 3.73^{*}$ & $13.90 \pm 6.21$ & 0.00 \\
\hline FPG (mmol/L) & $5.90 \pm 1.31^{\#}$ & $9.10 \pm 3.32^{\# \#}$ & 0.00 \\
\hline $\mathrm{CK}(\mathrm{U} / \mathrm{L})$ & $719 \pm 573$ & $583 \pm 560$ & 0.36 \\
\hline $\mathrm{TG}(\mathrm{mmol} / \mathrm{L})$ & $1.26 \pm 0.73$ & $1.67 \pm 0.98$ & 0.00 \\
\hline $\mathrm{TC}(\mathrm{mmol} / \mathrm{L})$ & $5.20 \pm 1.12$ & $5.30 \pm 1.40$ & 0.63 \\
\hline HDL-C (mmol/L) & $1.20 \pm 0.34$ & $1.09 \pm 0.29$ & 0.01 \\
\hline LDL-C (mmol/L) & $3.11 \pm 0.91$ & $3.18 \pm 1.08$ & 0.63 \\
\hline Creatinine $(\mu \mathrm{mol} / \mathrm{L})$ & $92 \pm 55$ & $120 \pm 106$ & 0.08 \\
\hline NSTEMI & $56(33.73 \%)$ & $41(47.12 \%)$ & 0.04 \\
\hline Conservative therapy & $106(63.86 \%)$ & $63(72.41 \%)$ & 0.17 \\
\hline Primary PCI & $55(33.13 \%)$ & $24(27.59 \%)$ & 0.37 \\
\hline Malignant arrhythmia & $19(11.45 \%)$ & $2(2.30 \%)$ & 0.01 \\
\hline Cardiac shock & $34(20.48 \%)$ & $15(17.24 \%)$ & 0.54 \\
\hline Killip classes III-IV & $51(30.72 \%)$ & $33(37.93 \%)$ & 0.25 \\
\hline Mortality rate & $20(12.05 \%)$ & $14(16.09 \%)$ & 0.37 \\
\hline Reinfarction rate & $6.02 \%$ & $13.79 \%$ & 0.04 \\
\hline Reinfarction interval (month) & $40 \pm 21$ & $18 \pm 16$ & 0.09 \\
\hline
\end{tabular}

Data were presented as mean \pm SD for normally distributed and continuous variables (Age, HR, SBP, DBP, APG, FPG, TC, HDL-C, and LDL-C) or median (IQR) for nonnormally distributed variables (CK, TG, creatinine, and reinfarction interval); categorical variables were reported as numbers and percentages. AMI: acute myocardial infarction; HR: heart rate; SBP: systolic blood pressure; DBP: diastolic blood pressure; APG: admission plasma glucose; FPG: fasting plasma glucose; CK: creatinine kinase; TG: triglyceride; TC: total cholesterol; HDL-C: high-density lipoprotein cholesterol; LDL-C: low-density lipoprotein cholesterol; NSTEMI: non-ST-segment elevation myocardial infarction; and PCI: percutaneous coronary intervention.

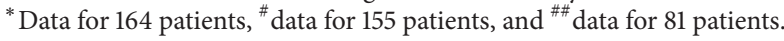

TABLE 2: Comparison of different admission glucose levels between nondiabetic group and diabetic group.

\begin{tabular}{|c|c|c|c|c|c|c|}
\hline \multirow{2}{*}{ Variable } & \multicolumn{2}{|c|}{ Nondiabetic group (mmol/L) } & \multirow{2}{*}{$P$} & \multicolumn{2}{|c|}{ Diabetic group $(\mathrm{mmol} / \mathrm{L})$} & \multirow{2}{*}{$P$} \\
\hline & $<11.1$ & $\geq 11.1$ & & $<11.1$ & $\geq 11.1$ & \\
\hline Cases & 140 & 24 & & 27 & 60 & \\
\hline Mortality rate & $11(7.86 \%)$ & $7(29.17 \%)$ & 0.006 & $44(14.81 \%)$ & $10(16.67 \%)$ & 1.000 \\
\hline Painless AMI & $27(19.29 \%)$ & $8(33.33 \%)$ & 0.121 & $6(22.22 \%)$ & $24(40.00 \%)$ & 0.107 \\
\hline TG & $1.26 \pm 0.81$ & $1.12 \pm 0.76$ & 0.409 & $1.79 \pm 1.21$ & $1.62 \pm 1.13$ & 0.533 \\
\hline HDL-C & $1.20 \pm 0.34$ & $1.20 \pm 0.30$ & 0.621 & $1.01 \pm 0.25$ & $1.13 \pm 0.30$ & 0.177 \\
\hline Creatinine & $83.62 \pm 57.20$ & $96.04 \pm 36.30$ & 0.013 & $102.53 \pm 77.26$ & $97.5 \pm 66.27$ & 0.690 \\
\hline NSTEMI & 47 (33.57\%) & $9(37.50 \%)$ & 0.708 & $13(48.15 \%)$ & $28(46.67 \%)$ & 0.898 \\
\hline Cardiac shock & $24(17.14 \%)$ & $8(33.33 \%)$ & 0.116 & $3(11.11 \%)$ & $12(20.00 \%)$ & 0.479 \\
\hline Killip classes (III-IV) & $38(27.14 \%)$ & $11(45.83 \%)$ & 0.065 & $9(33.33 \%)$ & $24(40.00 \%)$ & 0.553 \\
\hline Malignant arrhythmia & $19(13.57 \%)$ & $5(20.83 \%)$ & 0.630 & $0(0.00 \%)$ & $2(1.67 \%)$ & 1.000 \\
\hline
\end{tabular}

hyperglycemia subgroup was $7.86 \%$ and $29.17 \%$, respectively $(P<0.01)$. Likewise, when subgrouped by FPG level, the in-hospital mortality rate was $4.32 \%$ and $43.75 \%$, respectively $(P<0.01)$. However, there was no significant difference between nonhyperglycemia subgroup and hyperglycemia subgroup in mortality rate in the diabetic group. Moreover, as shown in Table 3, in the nondiabetic group, the incidence of cardiac shock in hyperglycemia subgroup is much higher than nonhyperglycemia subgroup (12.95\% versus $56.25 \%, P<$ $0.001)$.

3.3. Predictors of In-Hospital Mortality. In order to analyze which factor was closely associated with in-hospital mortality, we did a multivariate logistic regression analysis to eliminate 
TABLE 3: Comparison of different fasting glucose levels between nondiabetic group and diabetic group.

\begin{tabular}{|c|c|c|c|c|c|c|}
\hline \multirow{2}{*}{ Variable } & \multicolumn{2}{|c|}{ Nondiabetic group (mmol/L) } & \multirow{2}{*}{$P$} & \multicolumn{2}{|c|}{ Diabetic group (mmol/L) } & \multirow{2}{*}{$P$} \\
\hline & $<7.0$ & $\geq 7.0$ & & $<7.0$ & $\geq 7.0$ & \\
\hline Cases & 139 & 16 & & 28 & 53 & \\
\hline Mortality & $6(4.32 \%)$ & $7(43.75 \%)$ & 0.000 & $4(14.29 \%)$ & $7(13.21 \%)$ & 1.000 \\
\hline Painless & $29(20.86 \%)$ & $3(18.75 \%)$ & 1.000 & $7(25.00 \%)$ & $20(37.74 \%)$ & 0.248 \\
\hline TG & $1.28 \pm 0.79$ & $0.98 \pm 0.58$ & 0.233 & $1.74 \pm 1.21$ & $1.65 \pm 1.06$ & 0.780 \\
\hline HDL-C & $1.20 \pm 0.34$ & $1.16 \pm 0.26$ & 0.792 & $1.04 \pm 0.26$ & $1.12 \pm 0.31$ & 0.549 \\
\hline Creatinine & $72.50 \pm 37.25$ & $63.00 \pm 54.00$ & 0.291 & $98.96 \pm 72.25$ & $100.02 \pm 71.20$ & 0.923 \\
\hline NSTEMI & $48(34.53 \%)$ & $6(37.5 \%)$ & 0.813 & $12(42.86 \%)$ & 27 (50.94\%) & 0.488 \\
\hline Cardiac shock & $18(12.95 \%)$ & $9(56.25 \%)$ & 0.000 & $2(7.14 \%)$ & $10(18.87 \%)$ & 0.278 \\
\hline Killip classes (III-IV) & $34(24.46 \%)$ & $6(37.50 \%)$ & 0.408 & $11(39.29 \%)$ & $18(33.96 \%)$ & 0.625 \\
\hline Malignant arrhythmia & $17(12.23 \%)$ & $3(18.75 \%)$ & 0.732 & $0(0.00 \%)$ & $1(1.89 \%)$ & 1.000 \\
\hline
\end{tabular}

the influence of confounding factors. Our results showed that independent predictors of in-hospital mortality for nondiabetic patients with AMI were FPG (OR: 2.014; 95\% CI: 1.296-3.131, $P<0.01$ ) and creatinine (OR: $1.011 ; 95 \% \mathrm{CI}$ : 1.004-1.017, $P<0.01$ ) (Table 4). However, the predictors of in-hospital mortality in diabetic group were age (OR: 1.160; 95\% CI: 1.004-1.342, $P<0.05$ ) and creatinine (OR: 1.007; 95\% CI: 1.001-1.014, $P<0.05$ ) (Table 5).

\section{Discussion}

Several previous studies have demonstrated that elevated APG and HbAlc were powerful predictors of mortality and increased risk of cardiovascular complications in AMI patients both with and without diabetes [13-16]. They found that hyperglycemia was associated with larger infarct size [17], lower left ventricular ejection fraction (LVEF) [18], and poor prognosis. Previous reports also suggested that glycemic status, which poses a risk for CVD, differed in male and female individuals. The reason could be a difference in the basic mechanism of carbohydrate metabolism, hormone, and insulin sensitivity. But there were limited data on FPG and cardiovascular prognoses of female patients with AMI. In the present study, we defined fasting hyperglycemia in acute myocardial infarction phase to be $\geq 7.0 \mathrm{mmol} / \mathrm{L}$ and admission hyperglycemia to be $\geq 11.1 \mathrm{mmol} / \mathrm{L}$. This glycaemia cutoff point was convergent with other authors' reports [19]. Moreover, this cutoff point was recommended by worldwide standards for a tool for carbohydrate metabolism disorder diagnosis [20]. Our study found that elevated FPG level in nondiabetic group was a strong and independent predictor of increased risk of mortality and cardiac shock in patients with AMI, and elevated APG level was closely associated with higher mortality rate and plasma creatinine level. But in diabetic group, we did not find this association. These results were in accordance with several previous studies including both men and women $[21,22]$.

In recent years, the importance of FPG in the prognosis of AMI was being gradually recognized. Suleiman et al. [21] reported a higher adjusted prevalence of in-hospital mortality among nondiabetic patients with elevated FPG, using a cutoff value of FPG $>6.1 \mathrm{mmol} / \mathrm{L}(110 \mathrm{mg} / \mathrm{dL})$ for

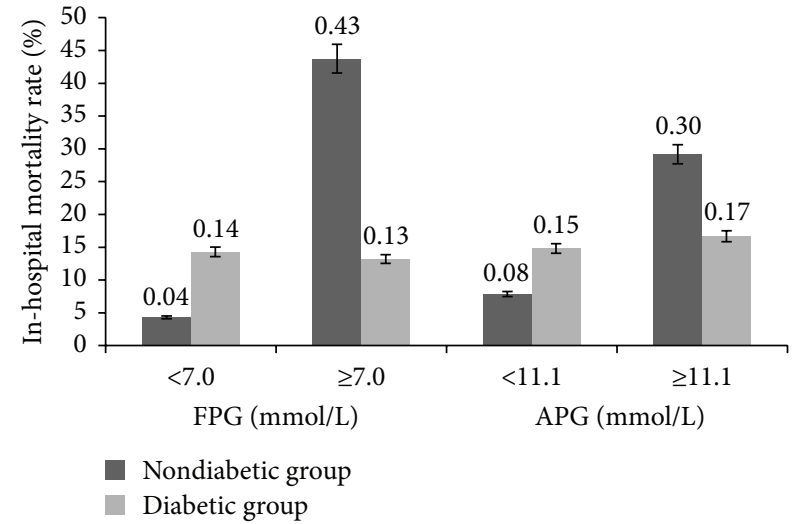

FIGURE 1: The relationship between blood glucose level and inhospital mortality rate.

fasting hyperglycemia. Compared with patients categorized as having normal FPG, the adjusted OR for 30-day mortality progressively increased with higher tertiles of elevated FPG (first tertile: 4.6 ; $95 \% \mathrm{CI}: 1.7$ to 12.7 ; second tertile: 6.4 ; 95\% CI: 2.5 to 16.6 ; and third tertile: 11.5 ; $95 \% \mathrm{CI}: 4.7$ to 20.0). Yang et al. [23] reported the FPG-stratified hazard ratios of in-hospital mortality in female patients of 1.037 (95\% CI: $0.820-2.262$ ) and 1.174 (95\% CI: 0.905-4.432) in mildly hyperglycemic and severely hyperglycemic group after multivariate adjustment. In our present study, we also found the prognostic value of APG in female nondiabetic patients with AMI.

T2DM is already an established risk factor for patients with AMI [24-26]. However, in contrast to these findings, we found higher in-hospital mortality of AMI in nondiabetic patients, contrary to the existing knowledge that the mortality of AMI was common mainly in diabetics. Moreover, our study demonstrated that elevated FPG and APG levels in nondiabetic group were strong and independent predictors of increased risk of mortality with AMI, but, in diabetic group, they were not. It showed that risk factors other than $\mathrm{DM}$ had a stronger association with the mortality of AMI in these cases. These results were consistent with the findings in some studies $[27,28]$. This phenomenon observed in the 


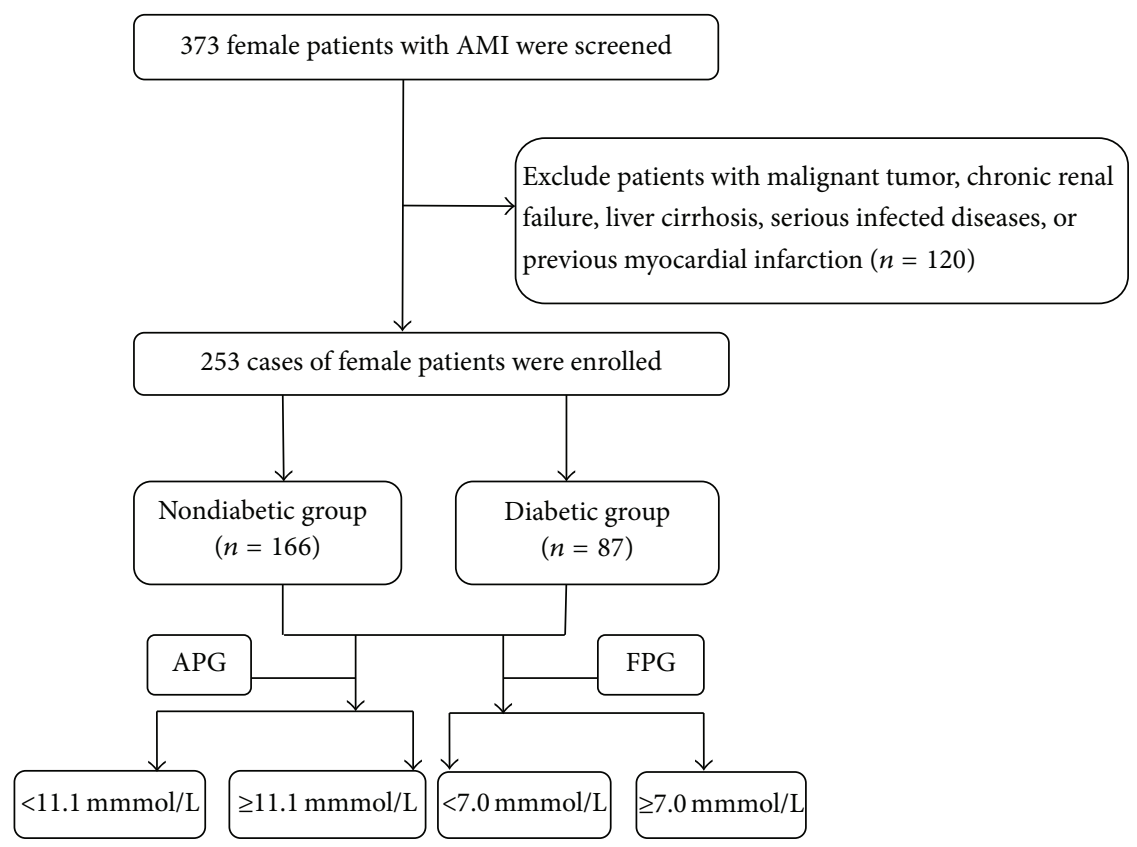

FIgURE 2: Flow chart of the inclusion of subjects in our study.

TABLE 4: Logistic regression analysis for mortality rate in nondiabetic group.

\begin{tabular}{|c|c|c|c|c|c|c|c|}
\hline \multirow{2}{*}{ Variable } & \multirow{2}{*}{$B$} & \multirow{2}{*}{ S.E. } & \multirow{2}{*}{ Wald } & \multirow{2}{*}{$P$} & \multirow{2}{*}{ OR } & \multicolumn{2}{|c|}{$95 \%$ CI for OR } \\
\hline & & & & & & Lower & Upper \\
\hline Age & 0.057 & 0.031 & 3.286 & 0.070 & 1.059 & 0.995 & 1.126 \\
\hline HR & -0.014 & 0.015 & 0.834 & 0.361 & 0.986 & 0.957 & 1.016 \\
\hline SBP & -0.027 & 0.019 & 1.999 & 0.157 & 0.973 & 0.937 & 1.011 \\
\hline DBP & -0.004 & 0.030 & 0.020 & 0.886 & 0.996 & 0.938 & 1.056 \\
\hline APG & 0.111 & 0.082 & 1.830 & 0.176 & 1.117 & 0.951 & 1.312 \\
\hline FPG & 0.700 & 0.225 & 9.672 & 0.002 & 2.014 & 1.296 & 3.131 \\
\hline TG & -0.272 & 0.605 & 0.203 & 0.652 & 0.762 & 0.233 & 2.491 \\
\hline $\mathrm{TC}$ & 0.050 & 0.674 & 0.006 & 0.940 & 1.052 & 0.281 & 3.939 \\
\hline HDL-C & -1.477 & 1.464 & 1.018 & 0.313 & 0.228 & 0.013 & 4.022 \\
\hline LDL-C & -0.097 & 0.769 & 0.016 & 0.900 & 0.908 & 0.201 & 4.096 \\
\hline $\mathrm{CK}$ & 0.000 & 0.000 & 1.094 & 0.296 & 1.000 & 0.999 & 1.000 \\
\hline Creatinine & 0.010 & 0.003 & 10.03 & 0.002 & 1.011 & 1.004 & 1.017 \\
\hline
\end{tabular}

current study may be a result of several factors, including improved treatment in the acute phase of AMI and increased long-term survival resulting from aggressive secondary prevention in diabetic patients [27]. Besides, we found that the frequency of malignant arrhythmia in the nondiabetic group was much higher than diabetes group, suggesting that malignant arrhythmia may contribute to narrowing the gap of short-term mortality between nondiabetic and diabetic patients. However, the underlying mechanisms are still unclear. Previous research indicated that hypoglycemia caused an acquired long QT syndrome and prolonged cardiac repolarization causes fatal cardiac arrhythmias [29]. But it did not fully explain these results. Besides, our study showed that the frequency of myocardial reinfarction was obviously higher in diabetic group, which indicated adverse longterm outcome in diabetic patients. Some studies also found that T2DM may abolish the beneficial effect of primary PCI on long-term risk of clinical reinfarction [30, 31]. For diabetic patients, undergoing primary PCI had the similar reinfarction rate compared with those who received the thrombolysis treatment [31]. Therefore, although T2DM did not impact short-term mortality rate, it still influenced the long-term mortality rate of AMI patients.

The relationship of the cause and effect between hyperglycemia and in-hospital mortality of AMI is still uncertain. On the one hand, serious AMI can cause stress hyperglycemia, resulting from a surge of stress hormones such as adrenaline, noradrenalin, and cortisol which induce or exacerbate an insulin-resistant state [32]. Relative insulin deficiency and excess catecholamine reduced glucose uptake by the ischemic myocardium and promoted lipolysis which increased circulating free fatty acids [21]. On the other hand, 
TABLE 5: Logistic regression analysis for mortality rate in diabetic group.

\begin{tabular}{lcccccc}
\hline Variable & $B$ & S.E. & Wald & $P$ & OR & \multicolumn{2}{c}{ 95\% CI for OR } \\
Lower & Upper \\
\hline Age & 0.149 & 0.074 & 4.044 & $\mathbf{0 . 0 4 4}$ & 1.160 & 1.004 \\
HR & 0.040 & 0.029 & 1.913 & 0.167 & 1.041 & 0.983 \\
SBP & -0.064 & 0.035 & 3.422 & 0.064 & 0.938 & 0.876 \\
DBP & -0.049 & 0.063 & 0.592 & 0.442 & 0.953 & 0.842 \\
APG & 0.122 & 0.090 & 1.827 & 0.176 & 1.129 & 0.947 \\
FPG & 0.025 & 0.139 & 0.032 & 0.858 & 1.025 & 0.781 \\
TG & 1.036 & 0.778 & 1.772 & 0.183 & 2.818 & 0.613 \\
TC & -1.449 & 1.557 & 0.866 & 0.352 & 0.235 & 0.011 \\
HDL-C & -0.237 & 2.890 & 0.007 & 0.935 & 0.789 & 1.004 \\
LDL-C & 1.749 & 1.575 & 1.233 & 0.267 & 5.748 & 0.003 \\
CK & 0.000 & 0.000 & 0.270 & 0.604 & 1.000 & 0.262 \\
Creatinine & 0.007 & 0.003 & 4.646 & $\mathbf{0 . 0 3 1}$ & 1.007 & 2.960 \\
\hline
\end{tabular}

hyperglycemia itself may lead to serious complications of AMI. Induction of endothelial dysfunction, oxidative stress, hypercoagulability, and impaired fibrinolysis may follow after hyperglycemia [21]. These factors ultimately produce vicious cycle. But some animal experiments indicated that short-term hyperglycemia may protest against ischemic myocardium [33, 34]. More researches will need to deeply uncover the mechanisms.

However, there are some reports contradicting our analysis and suggesting that the prognosis of diabetic patients may be significantly poor during the acute myocardial infarction phase. Moriyama et al. [35] presented a 2-fold higher hospital mortality rate in the subgroup of patients with DM and hyperglycemia in comparison to patients with DM without hyperglycemia. Likewise, Scuteri et al. [36] reported higher in-hospital mortality rate in the group of patients with DM and hyperglycemia. Multivariate analysis showed 5-fold risk of death in patients with hyperglycemia over $300 \mathrm{mg} / \mathrm{dL}$ and 2.8-fold in patients with hyperglycemia over $218 \mathrm{mg} / \mathrm{dL}$, in comparison to patients with blood glucose level below $161 \mathrm{mg} / \mathrm{dL}$.

In our analysis, the prognosis of patients without DM with concomitant hyperglycemia may, in part, be explained by the following differences in group characteristics. Firstly, patients with hyperglycemia were generally over 60 years, which agreed with a multivariate analysis showing that age was an independent factor of increased mortality rate in 1-year follow-up. In addition, nondiabetic patients with acute hyperglycemia showed an increased rate of inefficient fibrinolysis and the presence of multivessel coronary heart disease. These patients differed not only in the mentioned parameters but also in infarction severity including the concentration of myocardial necrotic markers and left ventricular ejection fraction from normal glucose patients. Hence, the importance of evaluation of plasma glucose during AMI for a better prognosis during follow-up period cannot be disregarded. However, we should realize that Blood glucose value (PFG and APG) is changeable in one day. Normally, the level of blood glucose changes within a limited range in nondiabetic patients. However, the inter- and intra-day glucose variability is relatively high in diabetic patients. In addition, blood glucose level is influenced by many other factors, such as diabetes status, serious complications and treatment. So before we analyse the association between hyperglycemia and complications of AMI, those factors should be took into consideration.

Several limitations of the research should be acknowledged. First, this was a single-center, nonrandomized, and retrospective study with a relatively small number of patients. Second, we did not evaluate long-term outcome of every AMI patient, so the relationship between FPG and long-term prognosis was not exactly assessed.

\section{Conflict of Interests}

The authors declare that they have no conflict of interests.

\section{Authors' Contribution}

Guojing Luo and Hong Liu contributed equally to this work.

\section{References}

[1] J. Marrugat, J. Sala, R. Masiá et al., "Mortality differences between men and women following first myocardial infarction," Journal of the American Medical Association, vol. 280, no. 16, pp. 1405-1409, 1998.

[2] M. Trappolini, F. M. Chillotti, R. Rinaldi et al., "Sex differences in incidence of mortality after acute myocardial infarction," Italian Heart Journal, vol. 3, no. 7, pp. 759-766, 2002.

[3] M. P. Savage, A. S. Krolewski, G. G. Kenien, M. P. Lebeis, A. R. Christlieb, and S. M. Lewis, "Acute myocardial infarction in diabetes mellitus and significance of congestive heart failure as a prognostic factor," The American Journal of Cardiology, vol. 62, no. 10, pp. 665-669, 1988.

[4] S. M. Donahoe, G. C. Stewart, C. H. McCabe et al., "Diabetes and mortality following acute coronary syndromes," Journal of the American Medical Association, vol. 298, no. 7, pp. 765-775, 2007. 
[5] P. C. Deedwania, M. I. Ahmed, M. A. Feller et al., "Impact of diabetes mellitus on outcomes in patients with acute myocardial infarction and systolic heart failure," European Journal of Heart Failure, vol. 13, no. 5, pp. 551-559, 2011.

[6] D. Aguilar, S. D. Solomon, L. Koøber et al., "Newly diagnosed and previously known diabetes mellitus and 1-year outcomes of acute myocardial infarction: the Valsartan in acute myocardial infarction (VALIANT) trial," Circulation, vol. 110, no. 12, pp. 1572-1578, 2004.

[7] M. Ishihara, S. Kojima, T. Sakamoto et al., "Acute hyperglycemia is associated with adverse outcome after acute myocardial infarction in the coronary intervention era," American Heart Journal, vol. 150, no. 4, pp. 814-820, 2005.

[8] M. Kosiborod, S. S. Rathore, S. E. Inzucchi et al., "Admission glucose and mortality in elderly patients hospitalized with acute myocardial infarction: implications for patients with and without recognized diabetes," Circulation, vol. 111, no. 23, pp. 3078-3086, 2005.

[9] M. Ishihara, "Acute hyperglycemia in patients with acute myocardial infarction," Circulation Journal, vol. 76, no. 3, pp. 563-571, 2012.

[10] M. Ishihara, S. Kojima, T. Sakamoto et al., " Comparison of blood glucose values on admission for acute myocardial infarction in patients with versus without diabetes mellitus," American Journal of Cardiology, vol. 104, no. 6, pp. 769-774, 2009.

[11] D. S. Pinto, A. H. Skolnick, A. J. Kirtane et al., "U-shaped relationship of blood glucose with adverse outcomes among patients with ST-segment elevation myocardial infarction," Journal of the American College of Cardiology, vol. 46, no. 1, pp. 178-180, 2005.

[12] M. S. Nieminen, M. Bohm, M. R. Cowie et al., "Executive summary of the guidelines on the diagnosis and treatment of acute heart failure," Italian Heart Journal, vol. 6, no. 4, pp. 218254, 2005.

[13] A. Dziewierz, D. Giszterowicz, Z. Siudak, T. Rakowski, J. S. Dubiel, and D. Dudek, "Admission glucose level and in-hospital outcomes in diabetic and non-diabetic patients with acute myocardial infarction," Clinical Research in Cardiology, vol. 99, no. 11, pp. 715-721, 2010.

[14] S. E. Capes, D. Hunt, K. Malmberg, and H. C. Gerstein, "Stress hyperglycaemia and increased risk of death after myocardial infarction in patients with and without diabetes: a systematic overview," The Lancet, vol. 355, no. 9206, pp. 773-778, 2000.

[15] I. B. Squire, C. P. Nelson, L. L. Ng, D. R. Jones, K. L. Woods, and P. C. Lambert, "Prognostic value of admission blood glucose concentration and diabetes diagnosis on survival after acute myocardial infarction: results from 4702 index cases in routine practice," Clinical Science, vol. 118, no. 8, pp. 527-535, 2010.

[16] J. R. Timmer, M. Hoekstra, M. W. N. Nijsten et al., "Prognostic value of admission glycosylated hemoglobin and glucose in nondiabetic patients with ST-segment-elevation myocardial infarction treated with percutaneous coronary intervention," Circulation, vol. 124, no. 6, pp. 704-711, 2011.

[17] I. Cruz-Gonzalez, S. Chia, O. C. Raffel et al., "Hyperglycemia on admission predicts larger infarct size in patients undergoing percutaneous coronary intervention for acute ST-segment elevation myocardial infarction," Diabetes Research and Clinical Practice, vol. 88, no. 1, pp. 97-102, 2010.

[18] D. E. Høfsten, B. B. Løgstrup, J. E. Møller, P. A. Pellikka, and K. Egstrup, "Abnormal glucose metabolism in acute myocardial infarction: influence on left ventricular function and prognosis," JACC: Cardiovascular Imaging, vol. 2, no. 5, pp. 592-599, 2009.

[19] E. J. Bakker, T. M. Valentijn, and K. M. van de Luijtgaarden, "Type 2 diabetes mellitus, independent of insulin use, is associated with an increased risk of cardiac complications after vascular surgery," Anaesth Intensive Care, vol. 41, no. 5, pp. 584590, 2013.

[20] P. Lugomirski, H. Guo, N. K. Boom, L. R. Donovan, D. T. Ko, and J. V. Tu, "Quality of diabetes and hyperlipidemia screening before a first myocardial infarction," Canadian Journal of Cardiology, vol. 29, no. 11, pp. 1382-1387, 2013.

[21] M. Suleiman, H. Hammerman, M. Boulos et al., "Fasting glucose is an important independent risk factor for 30-day mortality in patients with acute myocardial infarction: a prospective study," Circulation, vol. 111, no. 6, pp. 754-760, 2005.

[22] D. Aronson, H. Hammerman, M. R. Kapeliovich et al., "Fasting glucose in acute myocardial infarction: incremental value for long-term mortality and relationship with left ventricular systolic function," Diabetes Care, vol. 30, no. 4, pp. 960-966, 2007.

[23] S.-W. Yang, Y.-J. Zhou, X.-M. Nie et al., "Effect of abnormal fasting plasma glucose level on all-cause mortality in older patients with acute myocardial infarction: results from the Beijing Elderly Acute Myocardial Infarction Study (BEAMIS)," Mayo Clinic Proceedings, vol. 86, no. 2, pp. 94-104, 2011.

[24] S. M. Donahoe, G. C. Stewart, C. H. McCabe et al., "Diabetes and mortality following acute coronary syndromes," The Journal of the American Medical Association, vol. 298, no. 7, pp. 765-775, 2007.

[25] R. M. Jacoby and R. W. Nesto, "Acute myocardial infarction in the diabetic patient: pathophysiology, clinical course and prognosis," Journal of the American College of Cardiology, vol. 20, no. 3, pp. 736-744, 1992.

[26] K. H. Mak, D. J. Moliterno, C. B. Granger et al., "Influence of diabetes mellitus on clinical outcome in the thrombolytic era of acute myocardial infarction. GUSTO-I Investigators. Global utilization of streptokinase and tissue plasminogen activator for occluded coronary arteries," Journal of the American College of Cardiology, vol. 30, no. 1, pp. 171-179, 1997.

[27] H. L. Koek, S. S. Soedamah-Muthu, J. W. Kardaun et al., "Shortand long-term mortality after acute myocardial infarction: comparison of patients with and without diabetes mellitus," European Journal of Epidemiology, vol. 22, no. 12, pp. 883-888, 2007.

[28] S. T. Nauta, K. M. Akkerhuis, J. W. Deckers, and R. T. Van Domburg, "Short- and long-term mortality after myocardial infarction in patients with and without diabetes: changes from 1985 to 2008," Diabetes Care, vol. 35, no. 10, pp. 2043-2047, 2012.

[29] R. T. C. E. Robinson, N. D. Harris, R. H. Ireland, S. Lee, C. Newman, and S. R. Heller, "Mechanisms of abnormal cardiac repolarization during insulin-induced hypoglycemia," Diabetes, vol. 52, no. 6, pp. 1469-1474, 2003.

[30] H. D. White, H. R. Reynolds, A. C. Carvalho et al., "Reinfarction after percutaneous coronary intervention or medical management using the universal definition in patients with total occlusion after myocardial infarction: results from longterm follow-up of the Occluded Artery Trial (OAT) cohort," American Heart Journal, vol. 163, no. 4, pp. 563-571, 2012.

[31] M. M. Madsen, M. Busk, H. M. Søndergaard et al., "Does diabetes mellitus abolish the beneficial effect of primary coronary angioplasty on long-term risk of reinfarction after acute ST-segment elevation myocardial infarction compared with 
fibrinolysis? (A DANAMI-2 substudy)," The American Journal of Cardiology, vol. 96, no. 11, pp. 1469-1475, 2005.

[32] G. A. Oswald, C. C. T. Smith, D. J. Betteridge, and J. S. Yudkin, "Determinants and importance of stress hyperglycaemia in non-diabetic patients with myocardial infarction," British Medical Journal, vol. 293, no. 6552, pp. 917-922, 1986.

[33] L. M. Chu, R. M. Osipov, M. P. Robich et al., "Is hyperglycemia bad for the heart during acute ischemia?" Journal of Thoracic and Cardiovascular Surgery, vol. 140, no. 6, pp. 1345-1352, 2010.

[34] B. Rodrigues, D. M. T. Figueroa, J. Fang et al., "Short-term diabetes attenuates left ventricular dysfunction and mortality rates after myocardial infarction in rodents," Clinics, vol. 66, no. 8, pp. 1437-1442, 2011.

[35] N. Moriyama, M. Ishihara, T. Noguchi et al., "Admission hyperglycemia is an independent predictor of acute kidney injury in patients with acute myocardial infarction," Circulation Journal, vol. 78, no. 6, pp. 1475-1480, 2014.

[36] A. Scuteri, S. S. Najjar, M. Orru' et al., “Age- and genderspecific awareness, treatment, and control of cardiovascular risk factors and subclinical vascular lesions in a founder population: the SardiNIA Study," Nutrition, Metabolism and Cardiovascular Diseases, vol. 19, no. 8, pp. 532-541, 2009. 


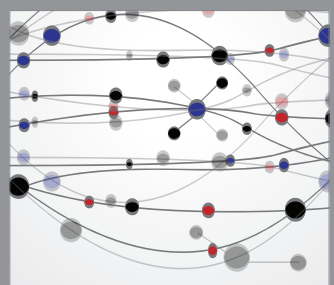

The Scientific World Journal
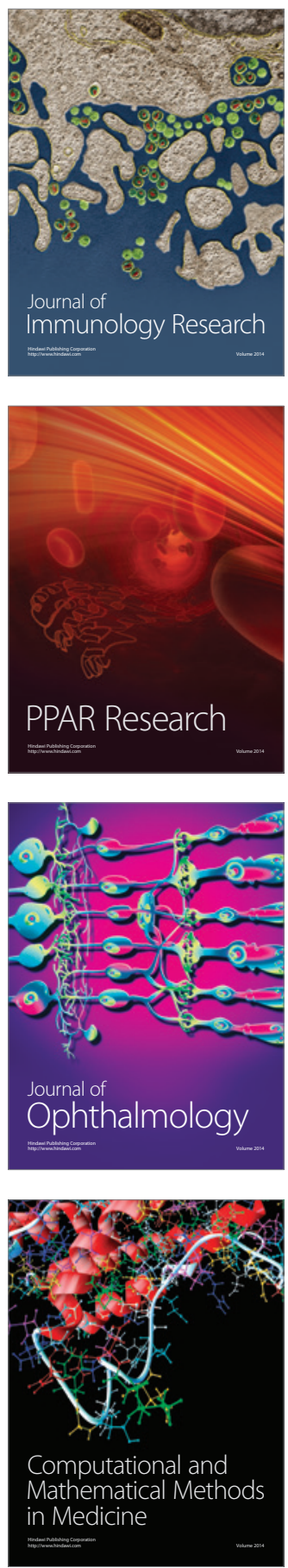

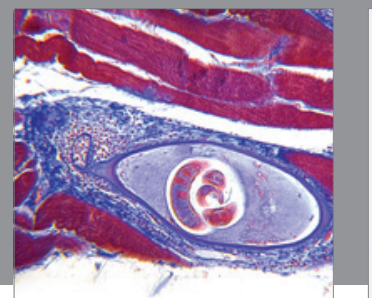

Gastroenterology

Research and Practice
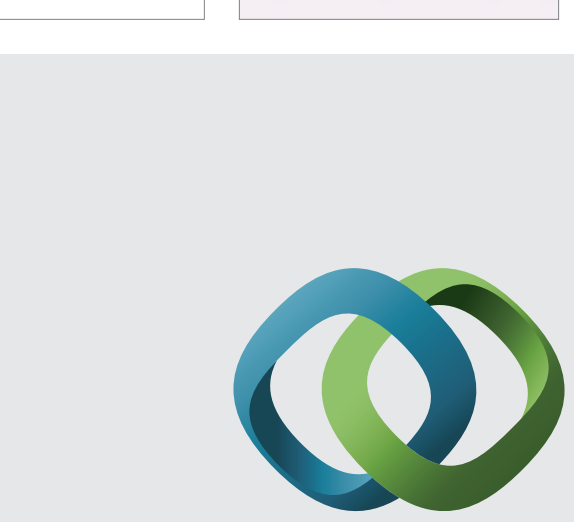

\section{Hindawi}

Submit your manuscripts at

http://www.hindawi.com
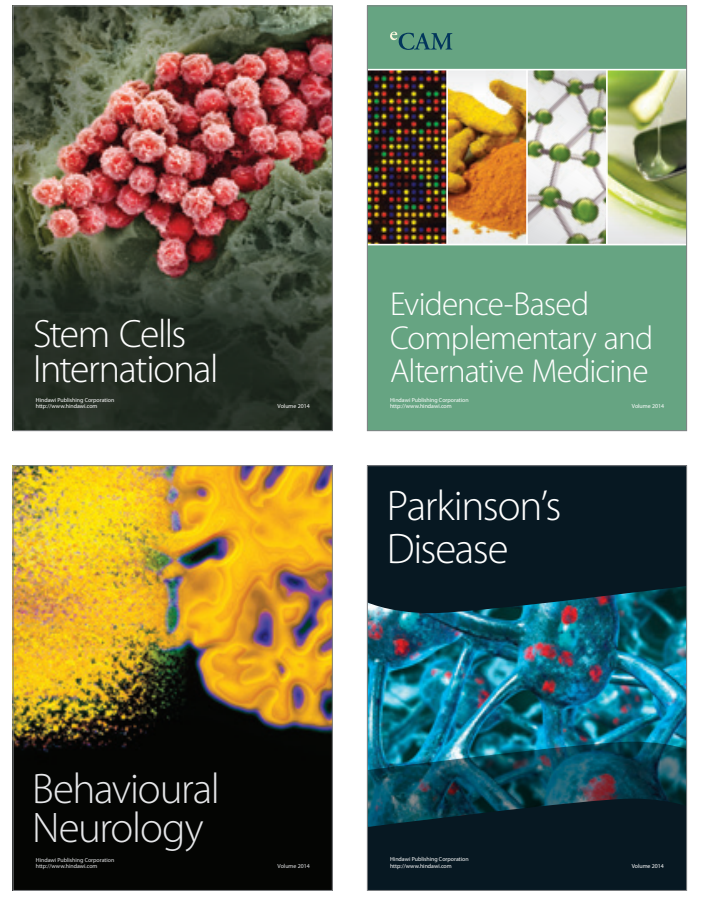
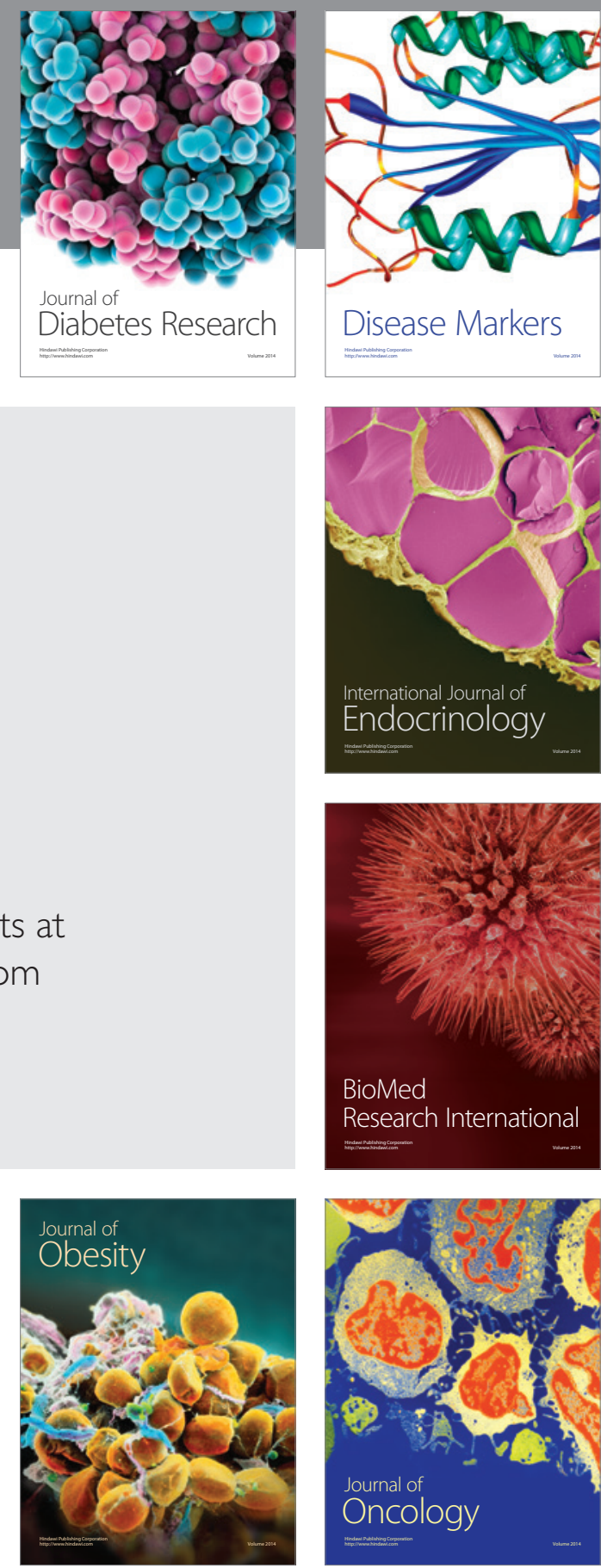

Disease Markers
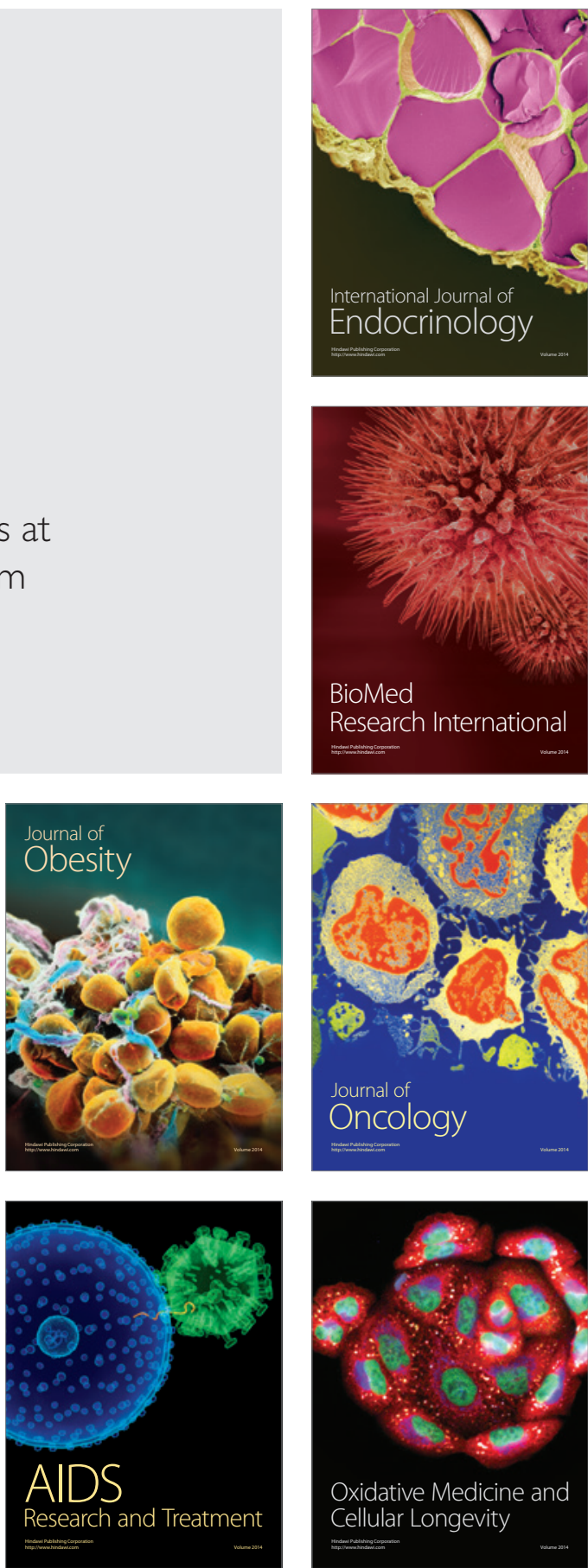